\title{
Summary of the working groups and suggestions for future research
}

\section{Introduction}

European Childhood Obesity Group (ECOG) was formed 20 years ago when a small group of practitioners working on obesity issues in Europe met to exchange views on childhood obesity and to develop a programme of action. Over the years, as the interest in this issue increased, the format of ECOG meetings evolved into a formal Congress, but this year to mark our 20-year anniversary ECOG went back to its 'workshop' roots to encourage a frank and free discussion and encourage ideas among participants from across the globe.

Four discussion topics were chosen and participants were allotted to specific groups for each discussion period. Topics discussed were:

1. Common approach and tools for paediatric obesity and prevention

2. Climate change and childhood obesity

3. Communication

4. Insulin resistance: early diagnosis, early treatment?

Groups were relatively small - fifteen to twenty - allowing opportunities for all who wished to participate in the discussions. Facilitators led dicussions and reported back in the main follow-up sessions. The reports below are drawn from the ideas pooled at the reporting back sessions. There is perhaps nothing startlingly novel here but who knows what seeds of discovery have been sown in the minds of the scientists and clinicians taking part in these discussions.

It should be noted that we refer widely to child obesity but we intend this term to include children significantly overweight since this deserves just as much attention as obesity itself and is potentially more rewarding to manage.

\section{Common approach and tools for paediatric obesity and prevention}

A 'healthy' environment not only helps prevent the development of child (and adult) obesity it also contributes to the treatment of obese patients since such an environment should help individuals avoid dietary temptation and encourage, or even facilitate, physical activity. However it is notable that these discussions did not formulate a specific definition nor diagnostic criteria for a 'healthy' environment.

We already know a lot about what should be done to prevent and treat obesity. What do we feel are the main factors preventing effective implementation of these procedures?
Lack of coordination between the different stakeholders working to reduce child obesity was seen as one of the most significant factors inhibiting effective implementation of policies. Part of the problem is that the treatment of obesity is predominantly hospital based and doctor led, while the prevention of obesity is usually community based and managed by many different professionals.

Effective implementation of prevention and treatment programmes for childhood obesity must give particular attention to the role of parents in modelling their children's behaviours. Approaches to families need to be positive and sensitive. Practices that promote healthy lifestyles for all the family should be established as early as possible in all children and therapeutic interventions for those with weight problems implemented as soon as a need is recognised, lest behaviours that tend to promote obesity become established.

\subsection{What are the roles of governments, public organisations, bealth-care systems and private bodies in implementing prevention and management of childbood obesity?}

All the aforementioned bodies have roles to play in the design and implementation of preventive and therapeutic interventions for childhood obesity but, as earlier indicated, their activities need to be more integrated.

For governments the role is primarily to implement actions that impact on the macro-environment. For instance, they are responsible for legislation relating to food advertising and food labelling; for facilitating improved access to healthy foods particularly in areas of social deprivation and disadvantage, for supporting initiatives leading to the consumption of more fruit and vegetables; and for creating and sustaining green areas and open spaces in towns and cities for children to play and exercise safely.

Health-care systems have big roles in improving the availability and accessibility of dedicated childhood obesity treatment centres and in ensuring that all staff in these centres have appropriate training. Government funding may be needed to provide for such centres' development.

Public organisations, such as scientific societies, are involved in the education and training of health-care professionals - paediatricians, community nurses, dietitians, psychologists and others - in the recognition, treatment and prevention of child (and adult) obesity.

Private bodies and organisations do not escape involvement. They are welcome to collaborate responsibly 
with governments, scientific societies and communities to promote better diets and more opportunities for healthy exercise and physical activity - in other words to help in making healthier choices possible for communities.

Much is expected of schools in the promotion of healthier environments. Paediatric dietetic advice may be needed to improve the quality of school food. All teachers, not just physical exercise (PE) teachers, can encourage activity amongst pupils. New schools can, if architects are aware of the need, be designed to facilitate physical movement amongst the pupils during the school day. To reach their goals, schools and other stakeholders (both governmental and private) need to provide information on their actions and the justification for these in ways readily comprehensible to both parents and children.

At local and national levels there should be a culture of providing information to communities about what constitutes a healthy lifestyle, promoting physical activity as well as committing to making healthy foods accessible and affordable to all. The involvement of professionals in giving advice and drawing up guidelines for communities should be seen as essential collaboration with other stakeholders.

\subsection{How do we evaluate effectiveness in the management and prevention of childbood obesity? Are there recognised criteria for 'success' in prevention or management? What should be considered 'success' and bow can we develop common methods of evaluation?}

There are no recognised criteria for 'success' in the management of childhood obesity and the scenario is even worse when trying to evaluate obesity prevention activities. A successful programme needs a follow-up that shows positive change in a broader range of health criteria than just BMI Z-score. Evidence for less specific changes to more healthy behaviours should be included since without such changes an individual's obesity might have increased further - it is impossible to know. Assessments of preventive and therapeutic projects are of little value until at least one year has lapsed since the intervention. Further, when assessing the success of therapeutic interventions, there must be some recognition of the severity of the obesity and the severity of any complication present. Far too many studies are too brief in duration, too small in subject numbers, too narrow in their observations of effect and too wide a range of ages, family background and degrees of obesity to allow easy interpretation of observed change.

\subsection{What are the research gaps in the area of management and prevention? What research should be developed to belp fill these gaps?}

There are still many gaps in our knowledge of nutritional status, food habits and physical activity among children of the European nations as well as gaps in knowledge of the different therapeutic approaches to child obesity across Europe.

Too many programmes still use very narrow approaches to the problem of childhood obesity. New research should build on existing data. A large comprehensive therapeutic programme that includes cognitive behavioural strategies to promote healthy lifestyles and weight changes was perceived as being potentially very useful.

There are major gaps in the training of health professionals in many areas relating to childhood nutrition but especially in the field of motivational and behavioural interview techniques. Programmes that attempt to elucidate those family behavioural issues that contribute to the development and continuation of child obesity deserve major promotion and support.

Research that makes governments aware of the costs of childhood obesity to both individuals and the state might stimulate governments to fund interventions leading to prevention and treatment.

\subsection{What should ECOG do in promoting prevention and management of childbood obesity?}

ECOG has a key role in the prevention and management of childhood obesity through its network of interested professionals established across Europe. It can create and/or facilitate links between stakeholders. It can contribute to the design of programmes at local, national and European level by discussing and suggesting food reformulation with the food industry. It can collaborate with the European Union (EU) and national institutions over developing food labelling that is comprehensible and helpful to consumers. It can provide guidelines for training professionals in good practice for the prevention and treatment of childhood obesity. It can even help with training professionals. Last, but not least, in order to do all this, ECOG should make itself more visible by responding to or promoting press conferences and by using a range of media to address both general public and politicians.

\section{Climate change and childhood obesity}

Research linking climate change and childhood obesity is only in its early stages but results so far suggest that there could be ecological associations between the two issues. Climate change could influence the risk of childhood obesity directly through reducing the energy expenditure required to maintain internal body temperature or indirectly through influencing social and environmental changes.

\subsection{Which issues relating to the relationship between climate change and obesity need action? Who should be responsible for effective changes in this area?}

The potential impact of climate change on both the individual and on groups - households and communities - deserves particular attention when considering the changing prevalence of obesity. Behaviours associated 
with the risk of obesity in westernised societies are also those thought to contribute to climate change. Examples are using motorised transport rather than walking or cycling; overheating homes; unnecessary and excessive purchasing of food; or wasting food due to lack of nutritional understanding and poor meal planning skills. Marketing strategies leading to excessive and unnecessary production of food are also at fault and have potentially adverse effects on the climate.

How much does the use of land for bio-fuel rather than for food production affect climate change and promote obesity? How much does the siting of energy generating solar panels on agricultural land affect the prevalence of obesity in children? Changes of this kind could have advantages and disadvantages. A reduction in the land area used for growing vegetables and other 'healthy' crops could raise prices and reinforce the socio-economic gap in childhood obesity prevalence. It could also lower the price of electricity and fuel for motor vehicles thus promoting more luxurious, obesity generating lifestyles.

Multidisciplinary research projects are needed to assess the impact of climate change on childhood nutrition with the European focus being overnutrition. Such multidisciplinary research should involve a variety of disciplines e.g. biochemistry, physiology, sociology, economics, agronomy, architecture, urban design and planning - if we are to understand the ecological links and ultimately persuade professionals to work together for positive change in this field.

This would seem to be an area where WHO, with its technical role in UNO, should take a lead. WHO can be aided by scientific groups with different expertise: agronomists, economists, doctors, city planners, etc. and, of course, governments.

\subsection{What is the role of medical scientists, especially those concerned about obesity, in influencing climate change? What can they do and bow sbould tbey do it?}

Climate change may seem remote from clinical and research medicine. Nevertheless doctors and clinical research scientists should feel involved in addressing the medical and biological consequences of rising environmental temperatures. One fruitful area of research might be exploration of changes in lipid metabolism in relation to climate and rising environmental temperatures. There may, for example, be value in investigating the role of brown and white adipose tissue metabolism. Further, the consequences of climate changes on levels and performance of physical activity should be addressed.

Clinicians and others involved in giving health advice should encourage safer and more intelligent use of foods by helping those who need advice on planning meals and budgeting; promoting the use of seasonal local vegetables; and recommending appropriate temperature levels in the home.
Most people are more concerned about the effect of climate change on themselves than about its global effects so all advice needs to link individuals' perceptions of climate change with issues relating to those individuals' own health. However, the attention of stakeholders such as politicians and those setting national and local community policies needs to be drawn to the links between climate change and health and the possible relationship with increasing childhood obesity.

\subsection{What are the gaps in research in relation to climate change and child nutrition? Are there research projects that could belp fill these gaps?}

Information on possible biological mechanisms linking climate change and obesity is needed. Research in this area is largely nonexistent. The information that does exist needs collating and reviewing. After that, laboratory, biochemical and physiological studies can be initiated and linked up with relevant socio-economic, agronomic and ecological studies.

\subsection{Is there a role for ECOG in the climate change debate?}

ECOG should plan to collate data and then mediate with the different stakeholders so as to bring this topic into the public arena. At this stage of knowledge, it is too early to adopt a particular stance or to make recommendations but it is important that ECOG accepts the preliminary position of stimulating further research. The annual ECOG conference could provide a suitable forum at which to collaborate with agencies involved in climate change and to bring them together with other interested parties.

\section{Communication}

\subsection{Communication is something that is taught extensively to doctors today. How is communication with obese children and their carers special?}

Communication with those around obese children must be alert to the sensitivities of the children and families involved. A positive approach should be adopted with blame centred on the environment rather than the family. But how is all this done? Too often, old one-way, communication models and strategies are the ones used when advising on the prevention and treatment of childhood obesity. On the whole, such methods have been shown to be somewhat ineffective. We need to conceive, develop and use novel ways of communication and encourage medical professionals to trial these in clinical practice. Effective communication is especially important in childhood obesity since communication needs to adapt not only to the subject's age and the family's ability to listen and understand but also to introduce the complex, 
multifaceted and not always attractive changes necessary to prevent and treat childhood obesity.

The success of any communication in meeting the knowledge and strategic needs of subjects, whether these are individuals or community groups, should always be evaluated with scientific objectivity and the results fed back to the communicators. Those involved with obesity in children (and adults) should be more critical of their own communication methodology and skills. Too often communication is considered successfully accomplished since the subjects have been 'told' about the issue. Too rarely do communicators ask themselves: 'have people got the message?', or 'are they implementing advice? If not, why not?' Medical professionals are taught to listen as well as speak but the need to listen is frequently overlooked. Developing an appropriately respectful and not openly negative judgment of the child and his/her family is critical for creating an environment in which the recipients of the advice are likely to absorb what is being said and then try to implement the advice. Once the right attitude and environment for communication have been developed, a variety of approaches can be tried but they must be two way allowing a circular model of health communication: health worker $\leftrightarrow$ patient $\leftrightarrow$ environment.

\subsection{How should bealth workers communicate with government, industry, media and the general public in order to promote interest and action for the benefit of obese children?}

The media provide opportunities for disseminating information about childhood obesity other than just face-to-face encounters. They can be used by all wishing to communicate although target audiences will differ according to who gives the message. Scientific societies such as ECOG can use modern media to reach specific groups or whole communities. Governments use media for promoting official guidelines and advice on health issues. Professionals experienced in communication techniques can be invaluable in developing strategies that have a chance of being effective since they will have experience with tested social marketing concepts and techniques.

Collaborations in child obesity management should not exclude private stakeholders who could promote healthier diets such as those working in the agricultural sector or those who might promote physical activity such as the manufacturers of trainers and those selling bicycles provided these stakeholders remain detached from selfpromotion.

As well as contributing to the prevention and management of childhood obesity the media can, and do, spread inaccurate information without good scientific foundation or advertise inappropriate advice on obesity management. ECOG believes strongly that all public statements that have no justification or sound scientific backing should be refuted assertively.
3.3 How do we assess and improve the impact of information for all the public, for example food labels, government nutrition policies and bealth recommendations?

Communicating nutritional advice is a complex matter. Simple messages or symbols to help people make healthy food choices have been proven to be effective when the local cultural background is taken into account. Thus simple food labelling methods such as the traffic light system, the Australian model of 'occasional food' and the keyhole system are claimed to have been evaluated successfully in systematic and objective ways. Hard scientific evidence for a methodology should have the effect of reducing adverse lobbying by the food industry.

\subsection{What is the role of ECOG in improving communication in relation to childhood obesity?}

ECOG should improve its website to include a site where there is advice on good and bad practice in the management of childhood obesity.

ECOG congresses should do more to promote ECOG's activities so as to publicise the organisation and its work. It has a key role to play in unifying and harmonising different stakeholders' communications. Messages from different sources that seem to give conflicting advice are too often the reasons given by members of the public to explain why they do not adopt or implement particular health advice.

ECOG should also schedule press conferences for appropriate stakeholders in order to promote valuable new research, projects and activities from within ECOG and from other scientific societies and their partners.

\section{Insulin resistance: early diagnosis, early treatment}

Cardio-metabolic risk factors can be detected in obese children and adolescents, so increasing early obesity is likely to be contributing increasingly to the prevalence of adult CVD. Cardiorespiratory fitness (CRF) is a health marker across the lifespan and the levels of CRF are inversely related to the presence of cardiovascular risk factors even in young people. To some extent CRF attenuates the detrimental effects of excess body fat.

\subsection{Can we summarise the relationship between adipokines, insulin and obesity?}

Adipose tissue is a rich source of many of the immunerelated mediators (e.g. cytokines, such as tumor-necrosis factor-alpha (TNF- $\alpha$ ) and interleukin-6 (IL-6)) involved in the inflammatory response and mediating cardiometabolic disorders. Adipose tissue, in addition to regulating fat mass and energy homeostasis, releases a large number of bioactive mediators such as the adipokines leptin, adiponectin and peptide YY, which modulate appetite and metabolism as well as inflammatory processes. 
Do we know enough about whether their effects are positive or negative and under what circumstances?

There are few studies that indicate how relevant any of these factors are in relation to the various clinical risk factors in childhood obesity. How are they affected or influenced by genetic make-up, age, sex and maturation for example? Do we know the normal range for many of these factors?

Insulin levels and insulin resistance are widely recognised as associated with complications in obesity but how much is hyperinsulinaemia the promoter of obesity or its consequence?

\subsection{What markers are useful indicators of risk or the presence of metabolic derangement? Do we bave any consensus on 'bealthy' levels for these criteria? Does age influence bow we interpret findings?}

It was the general view that history and clinical examination still have very significant roles in selecting those children likely to be at risk of metabolic derangement since these are easy non-invasive clinical processes. Metanalyses show an epidemiological link between obesity and weight excess during pregnancy and the risk of future obesity in the offspring. Is this an insulin dependent effect? The role of insulin and insulin growth factors on the development of adipose tissue and the interaction with adipokines (leptin, adiponectin) have been studied extensively in animal models and adult man but studies in pregnant women and children are far fewer. The roles of other factors such as sex binding hormones and vitamin D levels in influencing, or being markers of, the development of obesity complications remain unclear and need further investigation.

In clinical practice new anthropometric measures such as nuchal thickness and neck circumference are potential markers for linking metabolic derangement with the clinical picture but, again, these parameters need further study to see how they vary with age, sex and maturity as well in their links with the complications of obesity.

\subsection{Is 'metabolic syndrome' something for which we have well-defined criteria in childbood? Is metabolic syndrome a useful diagnosis in childbood? If not, what are the metabolic indicators that should cause concern in childbood?}

The aggregation of multiple CVD risk factors has been called the metabolic syndrome (MetS). Abdominal obesity, hyperglycaemia, dyslipidaemia and hypertension are commonly used to define the MetS. Several of the patho-physiological features associated with MetS in adults is not uncommon in children but MetS remains a combination of risk factors not a single metabolic pathway. How much does each risk factor act by simple addition to risk and how much do combinations of risk factors compound the overall risk? This is not known.

The contribution of insulin resistance to each of the MetS components is uneven and sometimes ill defined. Lack of any consistent definition (there seem to be at least eleven different definitions for MetS in childhood) gives the diagnosis of 'metabolic syndrome' very nonspecific value in clinical practice or epidemiological studies. New possible indicators of cardiometabolic complications - C-reactive protein (CRP) and neutrophile counts for example - are not included in the 'classic' MetS. The overall opinion was that a diagnosis of MetS did not advance clinical management although the detection of a number of risk markers should stimulate vigorous intervention.

\subsection{What are the main areas where there are gaps in the research? Can we suggest how the gaps can be explored by research projects?}

More use needs to be made of the results of large cohort studies (e.g. STYJOBS, IDEFICS). To reduce atherosclerosis, type 2 diabetes and the MetS interventions to prevent and treat obesity in childhood are necessary since there is clear tracking of CVD risk factors track towards adulthood.

Knowledge of what leads not only to risky levels of biological markers but to recognising when and how to implement programmes to avoid future disease is largely lacking. Healthy lifestyle programmes should focus on three areas of concern: diet (including food habits, eating behaviour and nutritional education); physical activity (including the type, intensity and duration, sedentariness such as time spent in watching television, playing video-games, sleeping) and the individual's psychosocial background. How do such programmes affect the levels of risk markers?

\subsection{Is there a specific role for ECOG in this area?}

ECOG recommends that existing large cohort studies should be used to assess the validity and limitation of epidemiological tools. These can lead to the incorporation of new tools into existing lists of criteria for risk of complications.

There is a need to develop research questionnaires that facilitate recognition of the precursors to insulin resistance, for example investigating the significance of short sleep duration. The whole field of the value of the various metabolic derangements in predicting further complications needs more scientific study. How much are these markers signs of the severity of the obesity in those genetically at risk and how much are they independent indicators of developing problems?

Evaluations of the nutritional status of children to include searching for markers of risk for, adolescents and even their parents/caregivers could lead to implementing appropriate intervention programmes. However these programmes need to be acceptable to the subjects and to be assessed for efficacy. The consensus view is that such programmes need to be multidisciplinary and multifaceted rather than focused on diet or on activity (for example). Such programmes should, where possible, investigate the effect of interventions on metabolic factors as well as anthropometric factors to review whether changes in both these work in parallel or independently. 


\section{Conclusion}

Scientific investigation into childhood obesity remains a valuable and fruitful area for research with much knowledge still lacking. ECOG as a group of those working with childhood obesity needs to focus on how scientific research and clinical practice can be made into effective management programmes if the present epidemic of childhood obesity is to be contained.

There seem to be some overall aspirations for ECOG that come out of this resume.

Working with other stakeholders - governmental and non-governmental, public and private/commercial - ECOG should:

- provide a focus for scientific and clinical communications about childhood obesity;
- highlight the need for appropriate and effective communication between health professionals and obese children and their families;

- develop tools for programmes dealing with prevention and treatment of obesity and for evaluating projects, programmes and training;

- collate, review and evaluate research and from this suggest areas for future research arising from these reviews. Research needs to build on what has already been done;

- promote its position with governments, other scientific societies and among health-care professionals as well as with the media and the private sector.

Margherita Caroli, MD, PhD Marie-Laure Frelut, MD Elizabeth Poskitt, MD Andrea Vania, MD, PhD 